Bundesgesundheitsbl 2013 · 56:687-697

DOI 10.1007/s00103-012-1658-1

Online publiziert: 27. Mai 2013

(c) Springer-Verlag Berlin Heidelberg 2013

\section{Additional material online}

An English full-text version of this article is available at SpringerLink under supplementary material:

dx.doi.org/10.1007/s00103-012-1658-1

M. Haftenberger · D. Laußmann · U. Ellert · M. Kalcklösch · U. Langen · M. Schlaud • R. Schmitz $\cdot$ M. Thamm

Abteilung für Epidemiologie und Gesundheitsmonitoring, Robert Koch-Institut, Berlin

\title{
Prävalenz von Sensibilisierungen gegen Inhalations- und Nahrungsmittelallergene
}

\section{Ergebnisse der Studie zur Gesundheit Erwachsener in Deutschland (DEGS1)}

(2003-2006) wurde zudem gezeigt, dass $82,2 \%$ der Kinder mit einer einzigen Sensibilisierung und 53,1\% der Kinder mit mehr als einer Sensibilisierung keine atopischen Erkrankungen aufwiesen [5].

Anfang bis Mitte der 1990er-Jahre wurde das Auftreten von Sensibilisierungen in der erwachsenen Bevölkerung einzelner deutscher Städte in verschiedenen Studien untersucht $[6,7,8,9]$. Die Ergebnisse dieser Studien zeigten bei Einsatz der gleichen Tests auf spezifische IgE-Antikörper eine erhebliche Streuung der Prävalenz allergischer Sensibilisierungen in Abhängigkeit vom Geschlecht, Alter, Sozialstatus und von der Region (West- vs. Ostdeutschland). Außerdem scheint die Prävalenz der Sensibilisierungen ebenso wie die der Allergien zeitlichen Veränderungen zu unterliegen. Beim Vergleich von 3 bevölkerungsbezogenen Querschnittstudien in Kopenhagen (Dänemark) wurde gezeigt, dass die Prävalenz einer Sensibilisierung gegen mindestens eines von 19 Inhalationsallergenen bei den 40-Jährigen von $14,9 \%$ in den Jahren 1976-1977 auf $19,7 \%$ in den Jahren 1982-1984 zugenommen hatte. 1999-2001 lag die Prävalenz für eine Sensibilisierung mit 27,6\% noch höher [10]. In Deutschland wurde 1991-1992 die Prävalenz einer Sensibilisierung gegen häufig vorkommende Inhalationsallergene in 2 für die Erwachsenenbevölkerung im Alter von 25 bis 69 Jahren in West- und Ostdeutschland repräsentativen Stichproben mit einem Test auf häufig vorkommende Inhalationsallergene (SX1-Test) ermittelt [11]. 1991 waren 27,4\% der westdeutschen Erwachsenen und 1992 24,2\% der ostdeutschen Erwachsenen gegen mindestens eines der im Test enthaltenden Inhalationsallergene sensibilisiert [12]. Im Rahmen des BGS98 wurden für die deutsche Erwachsenenbevölkerung im Alter von 18 bis 79 Jahren repräsentative Daten zum Auftreten allergischer Sensibilisierungen mit dem gleichen Testverfahren ermittelt. Demnach lag die Prävalenz einer Sensibilisierung gegen mindestens eines der im Test enthaltenden Inhalationsallergene bei $30 \%$ [4]. Um aktuelle Aussagen zur Prävalenz von Sensibilisierungen treffen zu können, wurden im Rahmen der Studie zur Gesundheit Erwachsener in Deutschland (DEGS1) zwischen 2008 und 2011 repräsentative Daten $\mathrm{zu}$ Sensibilisierungen gegen insgesamt 50 Allergene und gegen Mischungen aus häufigen Inhalationsallergenen bzw. Gräserpollen (Screeningtests) in der deutschen erwachsenen Wohnbevölkerung erhoben. Ziel der vorliegenden Arbeit ist es, erste deskriptive Ergebnisse vorzustellen. Die Veränderung der Prävalenz von Sensibilisierungen gegen Inhalationsallergene zwischen 1998 und 2008-2011, gemessen mit dem SX1-Test, wird durch Vergleich der Ergebnisse des BGS98 mit den DEGS1-Ergebnissen beschrieben.

\section{Material und Methoden}

Die „Studie zur Gesundheit Erwachsener in Deutschland“ (DEGS) ist Be- 
Tab. 1 Tests auf spezifisches lgE im Serum in der „Studie zur Gesundheit Erwachsener in

Deutschland" (DEGS1)

Nahrungsmittelallergene

\begin{tabular}{|c|c|c|c|}
\hline f1 & Hühnereiweiß & & \\
\hline $\mathrm{f} 2$ & Milcheiweiß/Kuhmilch & \multicolumn{2}{|c|}{ Tierepithelien } \\
\hline $\mathrm{f} 4$ & Weizen/Weizenmehl & e1 & Katzenschuppen \\
\hline f5 & Roggen/Roggenmehl & e3 & Pferdeepithelien \\
\hline f6 & Gerste & e5 & Hundeschuppen \\
\hline f9 & Reis & \multicolumn{2}{|c|}{ Schimmelpilze } \\
\hline $\mathrm{f} 10$ & Sesam & $\mathrm{m} 2$ & Cladosporium herbarum \\
\hline $\mathrm{f13}$ & Erdnuss & $\mathrm{m} 3$ & Aspergillus fumigatus \\
\hline rf352 & Rekombinant Erdnuss, rAra h8 & $\mathrm{m} 6$ & Alternaria alternata \\
\hline f14 & Soja & \multicolumn{2}{|c|}{ Gräserpollen } \\
\hline rf353 & Rekombinant Soja, rGly m4 & g6 & Lieschgras \\
\hline $\mathrm{f} 17$ & Haselnuss & $g 12$ & Roggenpollen \\
\hline $\mathrm{f} 20$ & Mandel & \multicolumn{2}{|c|}{ Baumpollen } \\
\hline $\mathrm{f} 24$ & Krabbe & $\mathrm{t} 2$ & Erle \\
\hline $\mathrm{f} 25$ & Tomate & t3 & Birke \\
\hline $\mathrm{f} 31$ & Karotte & t4 & Hasel \\
\hline $\mathrm{f} 35$ & Kartoffel & $\mathrm{t} 25$ & Esche \\
\hline f44 & Erdbeere & t215 & Rekombinant Birke, rBet v1 \\
\hline f49 & Apfel & \multicolumn{2}{|c|}{ Kräuterpollen } \\
\hline f84 & Kiwi & w1 & Echte Ambrosia \\
\hline f85 & Sellerie & w2 & Ausdauernde Ambrosia \\
\hline $\mathrm{f} 242$ & Kirsche & w3 & Dreilappige Ambrosia \\
\hline f309 & Kichererbse & w230 & Hauptallergen Ambrosia, nAmb a1 \\
\hline f335 & Lupinensamen & w6 & Beifuß \\
\hline f351 & $\begin{array}{l}\text { Rekombinant Krabbe Tropomyosin } \\
\text { rPen a1 }\end{array}$ & w231 & Hauptallergen Beifuß, nArt v1 \\
\hline f419 & Pfirsich molekular, rPru p1 & \multicolumn{2}{|c|}{ Sonstige Allergene } \\
\hline \multicolumn{2}{|c|}{ Hausstaubmilben } & K82 & Latex \\
\hline d1 & Hausstaubmilbe d. pteronyssinus & Ro214 & $\begin{array}{l}\text { Cross-reactive-carbohydrate-determinant } \\
\text { (CCD) }\end{array}$ \\
\hline \multicolumn{2}{|c|}{ Insektengifte } & \multicolumn{2}{|c|}{ Allergenmischungen } \\
\hline i1 & Bienengift & SX1 & $\begin{array}{l}\text { Aeroallergene: } \mathrm{t} 3, \mathrm{~g} 6, \mathrm{~g} 12, \mathrm{w} 6, \mathrm{e} 1, \mathrm{e} 5 \text {, } \\
\mathrm{d} 1, \mathrm{~m} 2\end{array}$ \\
\hline i3 & Wespengift & GX1 & Gräsera: g3, g4, g5, g6, g8 \\
\hline
\end{tabular}

standteil des Gesundheitsmonitorings des Robert Koch-Instituts (RKI). Konzept und Design von DEGS sind an anderer Stelle ausführlich beschrieben [13, $14,15,16,17]$. Die erste Erhebungswelle (DEGS1) wurde von 2008 bis 2011 durchgeführt und umfasste Befragungen, Untersuchungen und Tests $[18,19]$. Zielpopulation war die in Deutschland lebende Bevölkerung im Alter von 18 bis 79 Jahren. DEGS1 hat ein Mischdesign, das gleichzeitig quer- und längsschnittliche Analysen ermöglicht. Hierbei wurde eine Einwohnermeldeamtsstichprobe gezogen, die ehemalige Teilnehmerinnen und Teilnehmer des Bun-
des-Gesundheitssurveys 1998 (BGS98) ergänzt. Insgesamt nahmen 8152 Personen teil, darunter 4193 Ersteingeladene (Response 42\%) und 3959 ehemalige Teilnehmerinnen und Teilnehmer des BGS98 (Response 62\%). 7238 Personen besuchten eines der 180 Untersuchungszentren, 914 wurden ausschließlich befragt. Die Nettostichprobe [17] ermöglicht für den Altersbereich von 18 bis 79 Jahren repräsentative Querschnittanalysen und Trendaussagen im Vergleich mit dem BGS98 ( $n=7988$, davon 7116 in Untersuchungszentren). Die Daten der erneut Teilnehmenden sind für Längsschnittanalysen nutzbar.
Während der körperlichen Untersuchung wurden eine Blut- und eine Urinprobe zur Labordiagnostik gewonnen. Zur Allergiediagnostik wurde ein quantitativer Nachweis spezifischer IgE-Antikörper im Serum durchgeführt. Dabei kam das Testsystem IMMUNOCAP der Firma Phadia (jetzt Thermo Fisher Scientific) zum Einsatz. Die Bestimmungen erfolgten auf dem Gerätesystem UNICAP 1000 (Phadia). Die Konzentrationsangaben der spezifischen IgE-Antikörper erfolgten in kU/l. Aus den Messwerten wurden 7 Klassen abgeleitet $(<0,35 \mathrm{kU} / \mathrm{l}$; 0,35-0,69 kU/l; 0,70-3,49 kU/1; 3,50$17,49 \mathrm{kU} / \mathrm{l} ; 17,50-49,99 \mathrm{kU} / \mathrm{l} ; 50,00-$ 99,99 kU/1; $\geq 100,00$ kU/l). Das Testergebnis wurde als positiv gewertet, wenn die Konzentration des spezifischen IgE einen Wert von $0,35 \mathrm{kU} / \mathrm{l}$ erreichte oder überschritt $(\geq 0,35 \mathrm{kU} / \mathrm{l})$. Insgesamt wurden 50 spezifische IgE-Antikörper bestimmt (- Tab. 1). Diese decken die wichtigsten Sensibilisierungen aus dem Innenraumbereich (Hausstaubmilbenkot, Tierepithelien und Schimmelpilze) und dem Außenbereich (Gräser-, Baum- und Kräuterpollen) sowie Sensibilisierungen gegen Nahrungsmittel und Insektengifte ab. Zusätzlich wurden 2 Screeningtests durchgeführt: der SX1-Test auf häufig vorkommende Inhalationsallergene [Hausstaubmilbe (d1), Birkenpollen (t3), Lieschgraspollen (g6), Roggenpollen (g12), Beifußpollen (w6), Katzenschuppen (e1), Hundeschuppen (e5) und Cladosporium herbarum (m2)] und der GX1-Test auf wichtige Gräserpollen [Knäuelgras (g3), Wiesenschwingel (g4), Lolch (g5), Lieschgras (g6) und Wiesen-Rispengras (g8)].

Zur Feststellung der zeitlichen Veränderungen bei der Prävalenz von Sensibilisierung gegen Inhalationsallergene wurden die diesbezüglichen in DEGS1 ermittelten Daten mit denen aus dem BGS98 verglichen unter der begründeten Annahme, dass sich der Test auf Inhalationsallergene (SX1) im Zeitraum zwischen beiden Untersuchungen nicht verändert hat (Mitteilung der Herstellerfirma). Der BGS98 wurde zwischen Oktober 1997 und März 1999 in einer repräsentativen Stichprobe der in Deutschland lebenden Wohnbevölkerung bei 7124 18- bis 79-Jährigen durchgeführt. Im BGS98 wurde zur Allergiediagnostik der gleiche Screeningtest auf In- 
halationsallergene (SX1) wie im DEGS1 eingesetzt [4].

Die für die vorliegende Auswertung relevanten soziodemografischen Merkmale wurden den Selbstausfüllfragebögen entnommen. Der Sozialstatus wurde anhand eines Indexes bestimmt, in den Angaben zu Schulbildung und beruflicher Ausbildung, beruflicher Stellung sowie Haushaltsnettoeinkommen (bedarfsgewichtet) eingehen und der eine Einteilung in eine niedrige, mittlere und hohe Statusgruppe ermöglicht [20]. Die regionale Zuordnung erfolgte nach Wohnort in Ost- (neue Bundesländer inklusive Berlin) und Westdeutschland (alte Bundesländer). Die Wohnregion West wurde weitergehend in Nordwest (Schleswig-Holstein, Hamburg, Bremen und Niedersachsen), Nordrhein-Westfalen, Mitte (Hessen, Saarland und Rheinland-Pfalz) und Süd (Bayern und Baden-Württemberg) stratifiziert. Die Stratifizierung nach Gemeindetyp erfolgte, wie in anderen Auswertungen zum Thema Allergien [3], nach Einwohneranzahl in den Klassen: ländlich bis unter 5000 Einwohner, kleinstädtisch 5000 bis unter 20.000 Einwohner, mittelstädtisch 20.000 bis unter 100.000 Einwohner und großstädtisch 100.000 Einwohner oder mehr.

Die Querschnitt- und Trendanalysen wurden mit einem Gewichtungsfaktor durchgeführt, der Abweichungen der Stichprobe von der Bevölkerungsstruktur (Stand 31.12.2010) hinsichtlich Alter, Geschlecht, Region und Staatsangehörigkeit sowie Gemeindetyp und Bildung korrigiert [17]. Für den Untersuchungsteil wurde ein gesonderter Gewichtungsfaktor berechnet. Bei der Berechnung der Gewichtung für die ehemaligen Teilnehmenden des BGS98 wurde die Wiederteilnahmewahrscheinlichkeit, basierend auf einem logistischen Modell, berücksichtigt. Für die Durchführung von Trendanalysen werden die Daten des BGS98 auf Bevölkerungsstand zum 31.12.2010 altersadjustiert. Eine Nonresponder-Analyse und der Vergleich einzelner erhobener Indikatoren mit Daten der amtlichen Statistik weisen auf eine hohe Repräsentativität der Stichprobe für die Wohnbevölkerung in Deutschland hin [17]. Um sowohl die Gewichtung als auch die Korrelation der Teilnehmenden innerhalb einer Gemein-

Bundesgesundheitsbl 2013 · 56:687-697 DOI 10.1007/s00103-012-1658-1

(c) Springer-Verlag Berlin Heidelberg 2013

\section{Haftenberger · D. Laußmann · U. Ellert · M. Kalcklösch · U. Langen · M. Schlaud · R. Schmitz $\cdot$ M. Thamm \\ Prävalenz von Sensibilisierungen gegen Inhalations- und Nahrungsmittelallergene. Ergebnisse der Studie zur Gesundheit Erwachsener in Deutschland (DEGS1)}

\section{Zusammenfassung}

Aufgrund der Zunahme von Allergien in Deutschland sind aktuelle Daten zur Prävalenz allergischer Sensibilisierungen von großem Interesse. Im Rahmen der ,Studie zur Gesundheit Erwachsener in Deutschland (DEGS1)" (2008-2011) wurden an einer bevölkerungsbezogenen Stichprobe von 7025 18- bis 79-Jährigen Blutproben auf spezifische lgE-Antikörper gegen 50 verbreitete Einzelallergene untersucht sowie 2 Tests mit jeweils einer Mischung aus Inhalationsallergenen (SX1) und Gräserpollen (GX1) durchgeführt. 48,6\% der Teilnehmer wiesen mindestens eine Sensibilisierung auf. Mehr Männer als Frauen waren gegen mindestens ein Allergen sensibilisiert. Zudem waren mehr jüngere als ältere Teilnehmer und mehr Personen mit höherem Sozialstatus von mindestens einer Sensibilisierung betroffen. Gegen Inhalationsallergene waren 33,6\% der Teilnehmer sensibilisiert, gegen Nahrungsmittelallergene $25,5 \%$ und gegen Insektengifte $22,5 \%$. Im Vergleich zum Bundes-Gesundheitssurvey 1998 (BGS98) hat die Prävalenz einer Sensibilisierung gegen Inhalationsallergene von $29,8 \%$ auf $33,6 \%$ zugenommen. Dieser Trend war nur bei Frauen signifikant. Die Ergebnisse des DEGS1 zeigen eine nach wie vor hohe Prävalenz allergischer Sensibilisierungen.

\section{Schlüsselwörter}

Allergische Sensibilisierung - Erwachsene . Bevölkerungsbezogen · Gesundheitssurvey

\section{Prevalence of sensitisation to aeraoallergens and food allergens. Results of the German Health Interview and Examination Survey for Adults (DEGS1)}

\section{Abstract}

In view of the increasing prevalence of allergies, up-to-date data on the prevalence of allergic sensitisation are of major interest. In the German Health Interview and Examination Survey for Adults (DEGS1) (2008-2011), blood samples from a population-based sample of 7,025 participants aged 18 to 79 years were analysed for specific lgE antibodies against 50 common single allergens and screened for common aeroallergens (SX1) and grass pollen (GX1). In all, $48.6 \%$ of the participants were sensitised to at least one allergen. Overall, men were more frequently sensitised to at least one allergen than women were. Sensitisations to at least one allergen were more common among younger than older participants and among participants with a higher socio-economic status. In all, $33.6 \%$ of the participants were sensitised to common aeroallergens, $25.5 \%$ to food allergens and $22.6 \%$ to wasp or bee venoms. Compared with the German National Health Interview and Examination Survey 1998 (GNHIES98), the prevalence of sensitisation to common aeroallergens increased from 29.8 to $33.6 \%$.This increase was statistically significant only in women. The results of DEGS1 still showed a high prevalence of allergic sensitisation. An English full-text version of this article is available at SpringerLink as supplemental.

\section{Keywords}

Allergic sensitization · Adults .

Population-based $\cdot$ Health survey de zu berücksichtigen, wurden die Konfidenzintervalle mit Verfahren für komplexe Stichproben mit dem Statistikpaket R Version 12.2 [21, 22, 23] unter Zugrundelegung der Beta-Verteilung [24, 25] bestimmt. Gruppenunterschiede wurden als statistisch signifikant angesehen, wenn sich die 95\%-Konfidenzintervalle nicht überschneiden.

\section{Ergebnisse}

Insgesamt konnte von 7025 der 7116 (98,7\%) befragten und untersuchten Teilnehmer des DEGS1 im Alter von 18 bis 79 Jahren eine Blutprobe auf spezifische IgE-Antikörper untersucht werden.

Fast die Hälfte dieser Teilnehmer $(48,6 \%)$ war gegen mindestens eines der 
Tab. 2 Prävalenz (in \%, gewichtet) und 95\%-Konfidenzintervall (95\%-KI) der allergischen Sensibilisierung in der deutschen erwachsenen Bevölkerung (DEGS1, n=7025) geschichtet nach Alter und Geschlecht

\begin{tabular}{|c|c|c|c|c|c|c|c|c|}
\hline \multirow{3}{*}{ 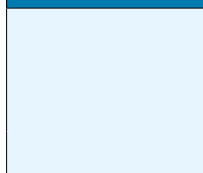 } & \multirow{3}{*}{$\begin{array}{l}\text { Ge- } \\
\text { schlecht }\end{array}$} & \multicolumn{7}{|l|}{ Altersgruppe } \\
\hline & & $\begin{array}{l}18 \text { bis } \\
29 \text { Jahre }\end{array}$ & $\begin{array}{l}30 \text { bis } \\
39 \text { Jahre }\end{array}$ & $\begin{array}{l}40 \text { bis } \\
49 \text { Jahre }\end{array}$ & $\begin{array}{l}50 \text { bis } \\
59 \text { Jahre }\end{array}$ & $\begin{array}{l}60 \text { bis } \\
69 \text { Jahre }\end{array}$ & $\begin{array}{l}70 \text { bis } \\
79 \text { Jahre }\end{array}$ & Gesamt \\
\hline & & $\%$ (95\%-KI) & $\%(95 \%-K I)$ & $\%(95 \%-K I)$ & $\%(95 \%-K I)$ & $\%(95 \%-K I)$ & $\%(95 \%-\mathrm{KI})$ & $\%(95 \%-K I)$ \\
\hline \multirow{3}{*}{$\begin{array}{l}\text { Mindestens } \\
\text { eine Sensibili- } \\
\text { sierung }\end{array}$} & Frauen & $55,0(50,2-59,7)$ & $50,9(45,0-56,8)$ & $46,5(41,7-51,4)$ & $\begin{array}{l}41,8 \\
(37,4-46,2) \\
\end{array}$ & $\begin{array}{l}36,1 \\
(31,6-40,6)\end{array}$ & $\begin{array}{l}39,3 \\
(34,2-44,5) \\
\end{array}$ & $45,3(43,3-47,3)$ \\
\hline & Männer & $55,2(49,4-60,8)$ & $60,3(54,0-66,4)$ & $55,0(49,6-60,4)$ & $\begin{array}{l}46,6 \\
(41,5-51,8)\end{array}$ & $\begin{array}{l}45,5 \\
(40,7-50,3)\end{array}$ & $\begin{array}{l}44,9 \\
(39,7-50,1)\end{array}$ & $51,8(49,6-54,1)$ \\
\hline & Gesamt & $55,1(51,6-58,6)$ & $55,7(51,2-60,2)$ & $50,9(47,1-54,6)$ & $\begin{array}{l}44,2 \\
(41,0-47,5)\end{array}$ & $\begin{array}{l}40,6 \\
(37,5-43,8)\end{array}$ & $\begin{array}{l}41,8 \\
(37,8-45,8) \\
\end{array}$ & $48,6(47,1-50,0)$ \\
\hline \multirow{3}{*}{$\begin{array}{l}\text { Sensibilisie- } \\
\text { rung gegen } \\
\text { inhalative } \\
\text { Allergene (SX1) }\end{array}$} & Frauen & $45,6(40,8-50,5)$ & $40,9(35,3-46,7)$ & $35,3(30,8-39,9)$ & $\begin{array}{l}26,8 \\
(22,7-31,1)\end{array}$ & $\begin{array}{l}20,9 \\
(17,1-25,1)\end{array}$ & $\begin{array}{l}18,0 \\
(13,7-23,0)\end{array}$ & $32,0(30,1-33,9)$ \\
\hline & Männer & $44,7(38,8-50,6)$ & $45,1(38,4-51,8)$ & $36,7(31,6-42,1)$ & $\begin{array}{l}31,5 \\
(27,1-36,1)\end{array}$ & $\begin{array}{l}25,4 \\
(21,7-29,4)\end{array}$ & $\begin{array}{l}21,3 \\
(17,0-26,2)\end{array}$ & $35,2(33,0-37,4)$ \\
\hline & Gesamt & $45,1(41,4-48,9)$ & $43,0(38,4-47,7)$ & $36,0(32,4-39,7)$ & $\begin{array}{l}29,1 \\
(26,2-32,1)\end{array}$ & $\begin{array}{l}23,1 \\
(20,5-25,9)\end{array}$ & $\begin{array}{l}19,5 \\
(16,5-22,8)\end{array}$ & $33,6(32,1-35,0)$ \\
\hline \multirow{3}{*}{$\begin{array}{l}\text { Sensibilisie- } \\
\text { rung gegen } \\
\text { Nahrungs- } \\
\text { mittel }\end{array}$} & Frauen & $28,4(24,2-32,9)$ & $28,3(23,2-33,9)$ & $26,3(22,1-30,9)$ & $\begin{array}{l}22,0 \\
(18,4-25,9)\end{array}$ & $\begin{array}{l}17,3 \\
(13,6-21,4)\end{array}$ & $\begin{array}{l}21,1 \\
(16,5-26,1)\end{array}$ & $24,2(22,3-26,1)$ \\
\hline & Männer & $29,8(25,0-34,9)$ & $31,8(27,2-36,8)$ & $30,4(25,6-35,5)$ & $\begin{array}{l}22,6 \\
(18,8-26,9)\end{array}$ & $\begin{array}{l}23,3 \\
(19,3-27,6)\end{array}$ & $\begin{array}{l}20,2 \\
(16,2-24,6)\end{array}$ & $26,9(25,0-28,8)$ \\
\hline & Gesamt & $29,1(25,9-32,5)$ & $30,1(26,6-33,9)$ & $28,4(25,2-31,9)$ & $\begin{array}{l}22,3 \\
(19,7-25,1)\end{array}$ & $\begin{array}{l}20,2 \\
(17,4-23,3)\end{array}$ & $\begin{array}{l}20,7 \\
(17,6-24,1)\end{array}$ & $26,9)$ \\
\hline \multirow{3}{*}{$\begin{array}{l}\text { Sensibilisie- } \\
\text { rung gegen } \\
\text { Insektengifte }\end{array}$} & Frauen & $18,4(14,8-22,3)$ & $20,2(15,4-25,6)$ & $18,8(14,9-23,1)$ & $\begin{array}{l}16,6 \\
(13,5-19,9)\end{array}$ & $\begin{array}{l}21,3 \\
(17,4-25,5)\end{array}$ & $\begin{array}{l}21,1 \\
(17,0-25,5)\end{array}$ & $1,1)$ \\
\hline & Männer & $21,8(17,2-26,9)$ & $25,9(20,6-31,7)$ & $29,5(25,1-34,2)$ & $\begin{array}{l}25,2 \\
(21,3-29,5) \\
\end{array}$ & $\begin{array}{l}24,7 \\
(20,5-29,3) \\
\end{array}$ & $\begin{array}{l}29,0 \\
(24,3-34,0) \\
\end{array}$ & 28,2) \\
\hline & Gesamt & $20,1(17,2-23,2)$ & $23,1(19,4$ & 24,3 & $\begin{array}{l}20,9 \\
(18,2-23,8)\end{array}$ & $\begin{array}{l}22,9 \\
(20,1-26,0)\end{array}$ & $\begin{array}{l}24,6 \\
(21,2-28,3)\end{array}$ & $4,2)$ \\
\hline \multirow{3}{*}{$\begin{array}{l}\text { Sensibilisie- } \\
\text { rung gegen } \\
\text { Gräserpollen }\end{array}$} & Frauen & $29,4(24,9-34,1)$ & $24,9(20,1-30,0)$ & $18,1(14,8-21,7)$ & $\begin{array}{l}9,8 \\
(7,6-12,4)\end{array}$ & $\begin{array}{l}10,2 \\
(7,6-13,4)\end{array}$ & $\begin{array}{l}6,6 \\
(4,5-9,0) \\
\end{array}$ & $8,4)$ \\
\hline & Männer & $32,8(27,8-38,1)$ & $31,4(25,9-37,2)$ & $23,9(19,8-28,3)$ & $\begin{array}{l}16,2 \\
(13,0-19,9)\end{array}$ & $\begin{array}{l}9,7 \\
(7,4-12,5)\end{array}$ & $\begin{array}{l}12,0 \\
(8,7-16,0)\end{array}$ & $20,3-23,8)$ \\
\hline & Gesamt & $31,1(27,9-34,5)$ & $28,2(24,3-32,3)$ & $21,1(18,5-23,7)$ & $\begin{array}{l}13,0 \\
(11,0-15,3)\end{array}$ & $\begin{array}{l}10,0 \\
(8,2-12,0)\end{array}$ & $\begin{array}{l}9,0 \\
(7,1-11,2)\end{array}$ & $18,3-20,6)$ \\
\hline \multirow{3}{*}{$\begin{array}{l}\text { Sensibilisie- } \\
\text { rung gegen } \\
\text { Baumpollen }\end{array}$} & Frauen & $24,2(20,6-28,0)$ & $22,7(18,2-27,6)$ & $21,8(17,9-26,0)$ & $\begin{array}{l}16,7 \\
(13,6-20,2)\end{array}$ & $\begin{array}{l}13,6 \\
(10,5-17,2)\end{array}$ & $\begin{array}{l}10,8 \\
(7,2-15,3)\end{array}$ & $18,7(17,2-20,3)$ \\
\hline & Männer & 22,9 & 24,8 & $9,7-28,6)$ & $\begin{array}{l}15,9 \\
(12,8-19,3)\end{array}$ & $\begin{array}{l}13,3 \\
(10,2-16,9)\end{array}$ & $\begin{array}{l}9,7 \\
(6,9-13,2)\end{array}$ & ,7-20,9) \\
\hline & Gesamt & $23,5(20,9-26,2)$ & $23,8(20,3-27,4)$ & $22,9(19,8-26,2)$ & $\begin{array}{l}16,3 \\
(14,1-18,7)\end{array}$ & $\begin{array}{l}13,5 \\
(11,2-16,0)\end{array}$ & $\begin{array}{l}10,3 \\
(8,0-13,0)\end{array}$ & $19,0(17,9-20,1)$ \\
\hline \multirow{3}{*}{$\begin{array}{l}\text { Sensibilisie- } \\
\text { rung gegen } \\
\text { Hausstaub- } \\
\text { milben }\end{array}$} & Frauen & $23,4(19,4-27,9)$ & $20,2(15,8-25,3)$ & $14,3(11,2-17,8)$ & $10,0(7,3-13,2)$ & $7,4(4,9-10,6)$ & $5,8(3,7-8,7)$ & $13,9(12,4-15,4)$ \\
\hline & Männer & $26,8(22,1-32,0)$ & $22,2(17,5-27,5)$ & $19,4(15,7-23,6)$ & $\begin{array}{l}13,4 \\
(10,2-17,2) \\
\end{array}$ & $\begin{array}{l}12,5 \\
(9,7-15,9)\end{array}$ & $\begin{array}{l}9,1 \\
(6,0-13,1)\end{array}$ & $18,0(16,3-19,8)$ \\
\hline & Gesamt & $25,2(21,8-28,8)$ & $21,3(17,9-24,9)$ & $16,9(14,6-19,5)$ & $11,7(9,7-14,0)$ & $9,9(8,1-11,9)$ & $7,3(5,4-9,6)$ & $15,9(14,8-17,1)$ \\
\hline \multirow{3}{*}{$\begin{array}{l}\text { Sensibilisie- } \\
\text { rung gegen } \\
\text { Kräuterpollen }\end{array}$} & Frauen & $18,4(14,8-22,5)$ & $13,3(9,2-18,3)$ & $10,3(7,7-13,4)$ & $5,5(3,9-7,5)$ & $5,0(3,3-7,2)$ & $5,5(3,3-8,3)$ & $9,9(8,6-11,3)$ \\
\hline & Männer & $17,4(13,8-21,4)$ & $15,4(11,6-19,7)$ & $15,5(12,2-19,1)$ & $7,9(5,8-10,4)$ & $5,8(3,9-8,1)$ & $10,5(7,5-14,1)$ & $12,5(11,2-13,9)$ \\
\hline & Gesamt & $17,9(15,3-20,7)$ & $14,4(11,4-17,6)$ & $12,9(10,7-15,4)$ & $6,7(5,2-8,4)$ & $5,4(4,1-6,9)$ & $7,7(5,8-10,0)$ & $11,2(10,3-12,2)$ \\
\hline \multirow{3}{*}{$\begin{array}{l}\text { Sensibilisie- } \\
\text { rung gegen } \\
\text { Tierepithelien }\end{array}$} & Frauen & $14,7(11,6-18,2)$ & $12,5(9,2-16,3)$ & $11,7(8,9-14,9)$ & $7,4(5,3-10,0)$ & $6,3(4,1-9,2)$ & $4,3(2,3-7,1)$ & $9,8(8,6-11,0)$ \\
\hline & Männer & $16,1(12,2-20,5)$ & $14,1(10,3-18,8)$ & $12,4(9,5-15,9)$ & $7,7(5,4-10,5)$ & $3,3(2,2-4,8)$ & $3,7(1,7-6,8)$ & $10,3(8,8-11,8)$ \\
\hline & Gesamt & $15,4(12,8-18,3)$ & $13,3(10,4-16,6)$ & $12,1(10,1-14,3)$ & $7,5(5,8-9,6)$ & $4,9(3,5-6,5)$ & $4,0(2,6-6,0)$ & $10,0(9,0-11,1)$ \\
\hline \multirow{3}{*}{$\begin{array}{l}\text { Sensibilisie- } \\
\text { rung gegen } \\
\text { Schimmelpilze }\end{array}$} & Frauen & $5,4(3,5-7,8)$ & $3,2(1,8-5,2)$ & $3,6(2,1-5,7)$ & $2,1(1,1-3,4)$ & $2,4(1,0-4,7)$ & $2,1(1,0-3,7)$ & $3,2(2,6-3,9)$ \\
\hline & Männer & $8,8(5,9-12,3)$ & $4,7(2,8-7,2)$ & $7,4(5,1-10,2)$ & $5,2(3,5-7,3)$ & $4,8(2,8-7,5)$ & $3,8(2,1-6,2)$ & $6,1(5,1-7,1)$ \\
\hline & Gesamt & $7,1(5,3-9,3)$ & $3,9(2,5-5,8)$ & $5,5(4,1-7,3)$ & $3,6(2,6-4,9)$ & $3,6(2,3-5,2)$ & $2,9(1,8-4,2)$ & $4,6(4,0-5,3)$ \\
\hline \multirow{3}{*}{$\begin{array}{l}\text { Sensibilisie- } \\
\text { rung gegen } \\
\text { Latex }\end{array}$} & Frauen & $4,4(2,8-6,5)$ & $6,8(3,9-10,9)$ & $4,0(2,4-6,2)$ & $3,1(2,0-4,7)$ & $2,0(0,9-4,0)$ & $2,8(1,2-5,3)$ & $3,9(3,1-4,7)$ \\
\hline & Männer & $6,6(4,5-9,2)$ & $6,5(4,1-9,7)$ & $6,5(4,2-9,6)$ & $2,5(1,5-3,9)$ & $2,9(1,6-4,6)$ & $4,3(2,5-6,9)$ & $5,0(4,2-6,0)$ \\
\hline & Gesamt & $5,5(4,1-7,2)$ & $6,7(4,7-9,1)$ & $5,3(3,9-7,1)$ & $2,8(2,0-3,8)$ & $2,4(1,5-3,7)$ & $3,5(2,2-5,1)$ & $4,4(3,9-5,1)$ \\
\hline
\end{tabular}




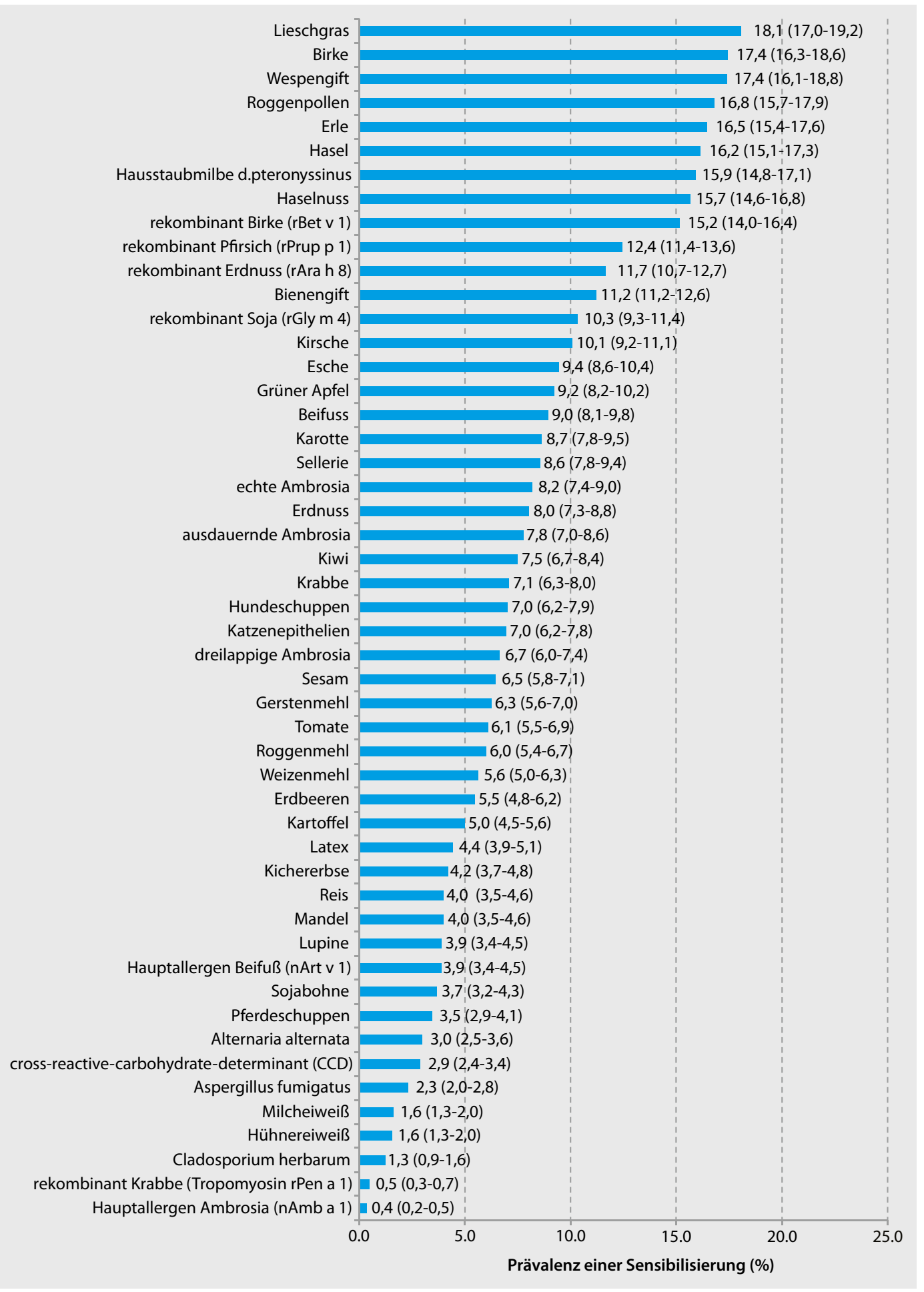

Abb. $1<$ Prävalenz (in \%, gewichtet) und 95\%-Konfidenzintervalle von Sensibilisierungen gegen 50 Allergene in der deutschen erwachsenen Bevölkerung (DEGS1, $\mathrm{n}=7025$ )

untersuchten Allergene sensibilisiert (- Tab. 2). Mehr als drei Viertel der sensibilisierten Personen (37,2\% aller Teilnehmer) wiesen mehr als eine Sensibilisierung auf. Lediglich 11,2\% waren nur gegen ein einziges Allergen sensibilisiert. Monosensibilisierungen lagen am häufigsten gegen Wespen- oder Bienengift vor. Die
Hälfte der Monosensibilisierungen bezog sich auf eine Sensibilisierung gegen Wespengift und fast $22 \%$ der Monosensibilisierungen gegen Bienengift.

- Abb. 1 zeigt die Häufigkeit der Sensibilisierung gegen die untersuchten Einzelallergene. $\mathrm{Zu}$ den 10 wichtigsten Einzelallergenen zählen die Gräserpollen
(Lieschgras und Roggenpollen), die meisten der untersuchten Baumpollen (Birke, Hauptallergen der Birke Bet vl, Erle und Hasel), Wespengift, Hausstaubmilbe und die Nahrungsmittelallergene Haselnuss und Pfirsich. Am seltensten wiesen die Teilnehmer Sensibilisierungen gegen das Krabbenallergen Tropomyosin Pen al, 
Tab. 3 Prävalenz (in \%, gewichtet) und 95\%-Konfidenzintervall (95\%-KI) der allergischen Sensibilisierung in der deutschen erwachsenen Bevölkerung (DEGS1, n=7025) geschichtet nach sozioökonomischem Status

\begin{tabular}{|c|c|c|c|c|}
\hline & \multirow{3}{*}{$\begin{array}{l}\text { Ge- } \\
\text { schlecht }\end{array}$} & \multicolumn{3}{|c|}{ Sozioökonomischer Status } \\
\hline & & Niedrig & Mittel & Hoch \\
\hline & & $\%(95 \%-K I)$ & $\%(95 \%-K I)$ & $\%(95 \%-K I)$ \\
\hline \multirow{3}{*}{$\begin{array}{l}\text { Mindestens eine Sensibi- } \\
\text { lisierung }\end{array}$} & Frauen & $39,4(34,7-44,2)$ & $45,0(42,4-47,6)$ & $53,4(48,5-58,2)$ \\
\hline & Männer & $49,0(43,8-54,1)$ & $50,9(47,7-54,0)$ & $56,5(52,2-60,7)$ \\
\hline & Gesamt & $44,1(40,6-47,6)$ & $47,8(45,9-49,7)$ & $55,1(51,8-58,3)$ \\
\hline \multirow{3}{*}{$\begin{array}{l}\text { Sensibilisierung gegen } \\
\text { inhalative Allergene (SX1) }\end{array}$} & Frauen & $22,7(18,8-27,0)$ & $32,2(29,8-34,7)$ & $41,5(36,8-46,3)$ \\
\hline & Männer & $26,9(22,8-31,3)$ & $35,1(32,2-38,0)$ & $42,4(38,2-46,6)$ \\
\hline & Gesamt & $24,8(22,0-27,7)$ & $33,6(31,8-35,4)$ & $42,0(39,0-45,0)$ \\
\hline \multirow{3}{*}{$\begin{array}{l}\text { Sensibilisierung gegen } \\
\text { Nahrungsmittel }\end{array}$} & Frauen & $18,6(15,1-22,3)$ & $24,6(22,3-27,0)$ & $28,9(24,8-33,3)$ \\
\hline & Männer & $23,0(19,2-27,2)$ & $26,1(23,3-28,9)$ & $32,6(29,0-36,3)$ \\
\hline & Gesamt & $20,7(18,1-23,6)$ & $25,3(23,6-27,1)$ & $30,9(28,2-33,8)$ \\
\hline \multirow{3}{*}{$\begin{array}{l}\text { Sensibilisierung gegen } \\
\text { Insektengifte }\end{array}$} & Frauen & $20,5(16,6-24,7)$ & $18,2(15,9-20,6)$ & $20,8(17,3-24,6)$ \\
\hline & Männer & $30,4(25,3-35,8)$ & $25,5(22,8-28,4)$ & $23,2(19,6-27,0)$ \\
\hline & Gesamt & $25,3(22,0-28,9)$ & $21,7(19,7-23,8)$ & $22,1(19,5-24,9)$ \\
\hline \multirow{3}{*}{$\begin{array}{l}\text { Sensibilisierung gegen } \\
\text { Gräserpollen }\end{array}$} & Frauen & $9,3(6,8-12,2)$ & $17,4(15,6-19,4)$ & $23,5(20,0-27,2)$ \\
\hline & Männer & $17,9(14,5-21,7)$ & $21,6(19,3-24,0)$ & $27,2(23,4-31,3)$ \\
\hline & Gesamt & $13,5(11,3-15,9)$ & $19,4(18,0-20,8)$ & $25,5(22,9-28,3)$ \\
\hline \multirow{3}{*}{$\begin{array}{l}\text { Sensibilisierung gegen } \\
\text { Baumpollen }\end{array}$} & Frauen & $11,7(8,7-15,2)$ & $19,1(17,1-21,2)$ & $25,1(21,1-29,4)$ \\
\hline & Männer & $15,1(11,8-18,8)$ & $19,0(16,8-21,3)$ & $24,2(21,1-27,4)$ \\
\hline & Gesamt & $13,4(11,0-16,0)$ & $19,1(17,7-20,5)$ & $24,6(22,0-27,3)$ \\
\hline \multirow{3}{*}{$\begin{array}{l}\text { Sensibilisierung gegen } \\
\text { Hausstaubmilben }\end{array}$} & Frauen & $11,3(8,6-14,5)$ & $13,8(12,0-15,9)$ & $16,1(12,6-20,0)$ \\
\hline & Männer & $12,6(9,5-16,1)$ & $17,8(15,5-20,3)$ & $22,3(19,1-25,8)$ \\
\hline & Gesamt & $11,9(9,8-14,3)$ & $15,8(14,3-17,3)$ & $19,5(17,0-22,2)$ \\
\hline \multirow{3}{*}{$\begin{array}{l}\text { Sensibilisierung gegen } \\
\text { Kräuterpollen }\end{array}$} & Frauen & $7,0(4,7-9,8)$ & $10,2(8,5-12,0)$ & $11,9(9,3-14,8)$ \\
\hline & Männer & $12,7(9,8-16,1)$ & $12,5(10,7-14,5)$ & $12,3(9,8-15,0)$ \\
\hline & Gesamt & $9,8(7,9-11,9)$ & $11,3(10,1-12,6)$ & $12,1(10,3-14,0)$ \\
\hline \multirow{3}{*}{$\begin{array}{l}\text { Sensibilisierung gegen } \\
\text { Tierepithelien }\end{array}$} & Frauen & $6,3(4,2-8,9)$ & $10,1(8,6-11,6)$ & $12,2(9,5-15,2)$ \\
\hline & Männer & $6,9(4,6-9,8)$ & $10,2(8,4-12,3)$ & $12,8(10,2-15,7)$ \\
\hline & Gesamt & $6,6(5,0-8,4)$ & $10,1(8,9-11,5)$ & $12,5(10,5-14,7)$ \\
\hline \multirow{3}{*}{$\begin{array}{l}\text { Sensibilisierung gegen } \\
\text { Schimmelpilze }\end{array}$} & Frauen & $2,1(1,1-3,4)$ & $3,0(2,2-4,0)$ & $5,3(3,7-7,2)$ \\
\hline & Männer & $4,1(2,4-6,4)$ & $6,5(5,2-7,9)$ & $6,3(4,5-8,3)$ \\
\hline & Gesamt & $3,1(2,1-4,3)$ & $4,7(3,9-5,5)$ & $5,8(4,6-7,2)$ \\
\hline \multirow{3}{*}{$\begin{array}{l}\text { Sensibilisierung gegen } \\
\text { Latex }\end{array}$} & Frauen & $3,2(1,7-5,4)$ & $4,0(3,1-5,2)$ & $3,5(2,2-5,2)$ \\
\hline & Männer & $5,7(3,5-8,6)$ & $5,3(4,1-6,7)$ & $3,7(2,6-5,2)$ \\
\hline & Gesamt & $4,4(3,1-6,1)$ & $4,6(3,9-5,5)$ & $3,6(2,8-4,7)$ \\
\hline
\end{tabular}

das Hauptallergen der Ambrosia ( $\mathrm{nAmb}$ a), den Schimmelpilz Cladosporium herbarum und gegen Milch- und Hühnereiweiß auf.

Eine detaillierte Beschreibung aller untersuchten Allergene nach soziodemografischen Merkmalen würde den Rahmen dieser Basispublikation sprengen. Deshalb erfolgt die Darstellung der Sensibilisierungsprävalenzen nur für den SX1Test und für Allergengruppen. Zur Klassifikation der Allergengruppen wurde die Zuordnung des Herstellers der eingesetzten Tests übernommen. Daraus resultier- tens eines der 26 getesteten Nahrungsmittelallergene auf. $\mathrm{Zu}$ den wichtigsten Nahrungsmittelallergenen zählten insbesondere die pollenassoziierten Nahrungsmittelallergene wie Haselnuss, Pfirsich, Soja und Erdnuss. Die meisten Frauen und Männer, die gegen Nahrungsmittelallergene sensibilisiert waren, wiesen auch Sensibilisierungen gegen Allergene der anderen Allergengruppen auf. Lediglich 1,7\% waren ausschließlich gegen Nahrungsmittelallergene sensibilisiert. Die Prävalenz einer Sensibilisierung gegen Insektengifte lag bei $22,6 \%$. Sensibilisierungen gegen Wespengift $(17,4 \%)$ traten häufiger auf als Sensibilisierungen gegen Bienengift (11,2\%). Gegen Gräser- und Baumpollen war jeweils fast ein Fünftel der Frauen und Männer sensibilisiert, gegen Kräuterpollen $11,2 \%$. Zu den Kräuterpollen gehören auch die Pollen der Gattung Ambrosia, die wegen der schnellen Verbreitung dieses invasiven Neophyten in einigen Regionen Deutschlands von großem Interesse sind. Die Prävalenz einer Sensibilisierung gegen Hausstaubmilben lag bei $15,9 \%$. Etwas geringer war die Prävalenz einer Sensibilisierung gegen Tierepithelien (10,0\%). Weniger als 5\% der Teilnehmer wiesen Sensibilisierungen gegen Schimmelpilzallergene $(4,6 \%)$ und gegen Latex auf $(4,4 \%)$.

\section{Soziodemografische Merkmale}

Für die meisten Allergengruppen lag die Prävalenz einer Sensibilisierung bei Männern höher als bei Frauen. Diese Unterschiede waren jedoch nur für die Sensibilisierungen gegen Insektengifte, Gräserpollen, Hausstaubmilben und Schimmelpilze statistisch signifikant und dies auch nicht in allen Altersgruppen (• Tab. 2).

Die Prävalenz einer Sensibilisierung gegen Insektengifte zeigte keine statistisch signifikanten Zusammenhänge mit dem Alter (• Tab. 2). Dagegen unterschied sich die Prävalenz der Sensibilisierungen bei den übrigen Allergengruppen und beim Test auf Inhalationsallergene statistisch signifikant zwischen den Altersgruppen mit signifikant häufigeren Sensibilisierungen bei jüngeren im Vergleich zu älteren Teilnehmern. Es zeigten sich jedoch für die verschiedenen Allergengruppen unterschiedliche Al- 
Tab. 4 Prävalenz (in \%, gewichtet) und 95\%-Konfidenzintervall (95\%-KI) der allergischen Sensibilisierung in der deutschen erwachsenen Bevölkerung (DEGS1, n=7025) geschichtet nach Region

\begin{tabular}{|c|c|c|c|c|c|c|c|}
\hline & \multirow{3}{*}{$\begin{array}{l}\text { Ge- } \\
\text { schlecht }\end{array}$} & \multicolumn{6}{|l|}{ Region } \\
\hline & & Ost & West & Nordwest & $\begin{array}{l}\text { Nordrhein-West- } \\
\text { falen }\end{array}$ & Mitte & Süd \\
\hline & & $\%(95 \%-K I)$ & $\%(95 \%-\mathrm{KI})$ & $\%(95 \%-K I)$ & $\%(95 \%-\mathrm{KI})$ & $\%(95 \%-K I)$ & $\%(95 \%-K I)$ \\
\hline \multirow{3}{*}{$\begin{array}{l}\text { Mindestens eine } \\
\text { Sensibilisierung }\end{array}$} & Frauen & $41,4(37,7-45,2)$ & $46,4(44,0-48,7)$ & $46,1(40,5-1,8)$ & $43,6(39,2-48,1)$ & $44,2(39,3-49,2)$ & $49,7(45,6-53,7)$ \\
\hline & Männer & $53,3(48,9-57,7)$ & $51,4(48,8-54,0)$ & $53,6(47,0-60,0)$ & $50,8(45,1-56,6)$ & $50,8(45,8-55,9)$ & $50,9(46,7-55,2)$ \\
\hline & Gesamt & $47,4(45,2-49,6)$ & $48,9(47,2-50,6)$ & $49,8(45,8-53,8)$ & $47,2(44,3-50,0)$ & $47,5(43,3-51,8)$ & $50,3(47,3-53,3)$ \\
\hline \multirow{3}{*}{$\begin{array}{l}\text { Sensibilisierung } \\
\text { gegen inhalative } \\
\text { Allergene (SX1) }\end{array}$} & Frauen & $26,6(22,8-30,8)$ & $33,4(31,3-35,5)$ & $32,9(27,3-38,9)$ & $34,7(30,5-39,1)$ & $33,5(28,6-38,7)$ & $32,5(29,2-35,9)$ \\
\hline & Männer & $32,9(28,9-37,2)$ & $35,8(33,3-38,3)$ & $39,3(31,7-47,3)$ & $37,2(33,2-41,5)$ & $37,1(30,8-43,6)$ & $32,1(28,5-35,9)$ \\
\hline & Gesamt & $29,8(27,3-32,3)$ & $34,6(32,9-36,3)$ & $36,1(31,4-41,0)$ & $36,0(33,4-38,6)$ & $35,3(30,5-40,3)$ & $32,3(29,8-34,9)$ \\
\hline \multirow{3}{*}{$\begin{array}{l}\text { Sensibilisierung } \\
\text { gegen Nahrungs- } \\
\text { mittel }\end{array}$} & Frauen & $21,9(18,9-25,1)$ & $24,8(22,5-27,1)$ & $25,3(19,1-32,3)$ & $25,3(21,5-29,4)$ & $23,2(18,2-28,8)$ & $24,8(21,2-28,6)$ \\
\hline & Männer & $26,9(23,6-30,4)$ & $26,9(24,7-29,2)$ & $25,9(20,9-31,3)$ & $27,2(22,2-32,6)$ & $27,9(23,4-32,7)$ & $26,8(23,4-30,4)$ \\
\hline & Gesamt & $24,4(22,1-26,8)$ & $25,8(24,3-27,4)$ & $25,6(21,6-29,9)$ & $26,3(23,0-29,7)$ & $25,5(21,9-29,5)$ & $25,8(23,5-28,2)$ \\
\hline \multirow{3}{*}{$\begin{array}{l}\text { Sensibilisierung } \\
\text { gegen Insekten- } \\
\text { gifte }\end{array}$} & Frauen & $21,4(17,9-25,1)$ & $18,6(16,5-20,9)$ & $15,9(12,3-20,0)$ & $12,4(9,4-15,8)$ & $17,5(13,4-22,1)$ & $25,5(21,7-29,6)$ \\
\hline & Männer & $31,5(27,2-36,0)$ & $24,5(22,1-27,0)$ & $20,4(16,4-24,8)$ & $21,1(17,0-25,6)$ & $23,2(18,5-28,5)$ & $30,1(25,5-35,0)$ \\
\hline & Gesamt & $26,4(23,7-29,3)$ & $21,5(19,6-23,5)$ & $18,1(15,2-21,3)$ & $16,7(14,1-19,5)$ & $20,4(17,2-23,8)$ & $27,8(24,1-31,7)$ \\
\hline \multirow{3}{*}{$\begin{array}{l}\text { Sensibilisierung } \\
\text { gegen Gräser- } \\
\text { pollen }\end{array}$} & Frauen & $15,4(12,3-19,0)$ & $17,3(15,6-19,0)$ & $17,8(14,2-21,8)$ & $16,7(13,5-20,2)$ & $18,2(14,9-21,9)$ & $16,9(13,8-20,4)$ \\
\hline & Männer & $20,1(16,9-23,6)$ & $22,5(20,5-24,6)$ & $23,0(17,4-29,4)$ & $23,4(20,5-26,4)$ & $23,0(18,5-27,9)$ & $21,3(17,9-25,0)$ \\
\hline & Gesamt & $17,8(15,7-20,0)$ & $19,9(18,5-21,3)$ & $20,4(16,8-24,3)$ & $20,0(17,8-22,4)$ & $20,6(17,5-24,0)$ & $19,1(16,9-21,5)$ \\
\hline \multirow{3}{*}{$\begin{array}{l}\text { Sensibilisierung } \\
\text { gegen Baum- } \\
\text { pollen }\end{array}$} & Frauen & $16,8(14,1-19,9)$ & $19,2(17,5-21,1)$ & $17,9(13,3-23,2)$ & $21,2(18,1-24,5)$ & $17,1(13,3-21,2)$ & $19,5(16,6-22,7)$ \\
\hline & Männer & $18,9(16,0-22,0)$ & $19,4(17,5-21,3)$ & $17,3(13,6-21,5)$ & $19,6(16,0-23,6)$ & $20,9(16,1-26,3)$ & $19,6(16,5-22,9)$ \\
\hline & Gesamt & $17,9(15,7-20,2)$ & $19,3(18,0-20,6)$ & $17,6(14,6-20,9)$ & $20,4(18,0-22,9)$ & $19,0(15,8-22,5)$ & $19,5(17,5-21,7)$ \\
\hline \multirow{3}{*}{$\begin{array}{l}\text { Sensibilisierung } \\
\text { gegen Hausstaub- } \\
\text { milben }\end{array}$} & Frauen & $10,7(8,2-13,5)$ & $14,7(13,1-16,5)$ & $14,0(10,8-17,6)$ & $16,9(13,4-20,8)$ & $15,8(11,3-21,3)$ & $12,9(10,3-15,9)$ \\
\hline & Männer & $15,5(12,5-18,8)$ & $18,7(16,7-20,8)$ & $21,6(16,5-27,4)$ & $22,5(19,1-26,1)$ & $14,6(8,8-22,4)$ & $16,1(13,4-19,1)$ \\
\hline & Gesamt & $13,1(11,2-15,1)$ & $16,7(15,4-18,1)$ & $17,7(14,6-21,3)$ & $19,6(17,4-22,1)$ & $15,2(11,3-19,9)$ & $14,5(12,7-16,4)$ \\
\hline \multirow{3}{*}{$\begin{array}{l}\text { Sensibilisierung } \\
\text { gegen Kräuter- } \\
\text { pollen }\end{array}$} & Frauen & $10,7(8,3-13,4)$ & $9,7(8,2-11,4)$ & $10,3(6,0-16,2)$ & $7,6(5,8-9,7)$ & $11,0(6,9-16,2)$ & $10,4(8,2-12,9)$ \\
\hline & Männer & $15,4(13,0-18,0)$ & $11,7(10,3-13,4)$ & $12,3(8,7-16,5)$ & $10,3(7,8-13,2)$ & $14,7(10,6-19,6)$ & $11,2(8,9-13,8)$ \\
\hline & Gesamt & $13,0(11,5-14,8)$ & $10,7(9,7-11,9)$ & $11,3(8,4-14,7)$ & $8,9(7,5-10,5)$ & $12,8(9,8-16,3)$ & $10,8(9,2-12,6)$ \\
\hline \multirow{3}{*}{$\begin{array}{l}\text { Sensibilisierung } \\
\text { gegen Tierepi- } \\
\text { thelien }\end{array}$} & Frauen & $9,0(7,0-11,2)$ & $10,0(8,7-11,4)$ & $10,3(7,4-13,7)$ & $10,3(7,9-13,1)$ & $10,2(7,4-13,5)$ & $9,5(7,2-12,2)$ \\
\hline & Männer & $9,2(7,0-11,7)$ & $10,6(8,9-12,4)$ & $12,3(7,8-18,0)$ & $12,4(9,2-16,1)$ & $10,1(6,1-15,3)$ & $8,4(6,2-11,0)$ \\
\hline & Gesamt & $9,1(7,5-10,8)$ & $10,3(9,1-11,5)$ & $11,3(8,0-15,2)$ & $11,3(9,2-13,7)$ & $10,1(7,2-13,7)$ & $8,9(7,3-10,8)$ \\
\hline \multirow{3}{*}{$\begin{array}{l}\text { Sensibilisierung } \\
\text { gegen Schimmel- } \\
\text { pilze }\end{array}$} & Frauen & $3,3(2,2-4,7)$ & $3,2(2,5-4,0)$ & $3,2(2,1-4,5)$ & $2,8(1,5-4,5)$ & $3,3(1,8-5,6)$ & $3,4(2,1-5,2)$ \\
\hline & Männer & $6,7(4,9-9,0)$ & $5,9(4,8-7,2)$ & $9,0(6,0-12,8)$ & $5,1(3,3-7,3)$ & $5,9(2,9-10,4)$ & $4,7(3,4-6,3)$ \\
\hline & Gesamt & $5,0(3,9-6,4)$ & $4,5(3,8-5,3)$ & $6,1(4,4-8,1)$ & $3,9(3,0-5,1)$ & $4,6(2,7-7,2)$ & $4,1(3,0-5,4)$ \\
\hline \multirow{3}{*}{$\begin{array}{l}\text { Sensibilisierung } \\
\text { gegen Latex }\end{array}$} & Frauen & $5,5(3,6-8,0)$ & $3,4(2,7-4,3)$ & $3,2(1,4-6,0)$ & $3,0(1,9-4,6)$ & $3,2(1,6-5,9)$ & $3,9(2,6-5,6)$ \\
\hline & Männer & $5,5(4,0-7,4)$ & $4,9(3,9-6,0)$ & $3,4(1,8-5,8)$ & $4,2(2,5-6,7)$ & $6,8(3,8-11,0)$ & $5,4(3,9-7,4)$ \\
\hline & Gesamt & $5,5(4,3-6,9)$ & $4,2(3,5-4,9)$ & $3,3(2,1-4,8)$ & $3,6(2,5-5,0)$ & $5,0(3,0-7,9)$ & $4,7(3,7-5,8)$ \\
\hline
\end{tabular}

tersgänge. Beispielsweise lag die Prävalenz der Sensibilisierungen gegen Nahrungsmittelallergene oder Latex bei Teilnehmern der Altersgruppen 18-29 Jahre bis 40-49 Jahre auf einem vergleichbaren Niveau, das signifikant höher war als die Prävalenz bei den Teilnehmern der älteren Altersgruppen, die sich untereinander nicht unterschieden. Die Prävalenz der Sensibilisierungen gegen Baumpollen unterschied sich nicht signifikant zwischen den Altersgruppen 18-29 Jahre bis 40-49 Jahre. Danach nahm die Prävalenz einer Sensibilisierung gegen
Baumpollen mit zunehmender Altersgruppe graduell ab. Die Prävalenz der Sensibilisierungen gegen Inhalationsallergene sowie die Prävalenzen in den Allergengruppen Gräserpollen, Hausstaubmilben und Tierepithelien nahmen mit zunehmender Altersgruppe graduell ab. Die Prävalenz der Sensibilisierungen gegen Kräuterpollen war in der jüngsten Altersgruppe am höchsten, nahm bis zum Alter von 60 bis 69 Jahren ab und danach wieder leicht zu. Sensibilisierungen gegen Schimmelpilze waren bei Teilnehmern der 3 ältesten Altersgruppen si- gnifikant seltener als in der Altersgruppe 18 bis 29 Jahre.

Teilnehmer mit hohem sozioökonomischem Status wiesen signifikant häufiger Sensibilisierungen gegen Inhalationsallergene, Nahrungsmittelallergene, Gräser- und Baumpollen, Hausstaubmilben, Schimmelpilze und Tierepithelien auf als Teilnehmer mit niedrigem sozioökonomischem Status (• Tab.3). Die Prävalenz einer Sensibilisierung gegen Insektengifte war unter Teilnehmern mit niedrigem sozioökonomischem Status etwas höher als bei Teilnehmern mit mittlerem 
Tab. 5 Prävalenz (in \%, gewichtet) und 95\%-Konfidenzintervall (95\%-KI) der allergischen Sensibilisierung in der deutschen erwachsenen Bevölkerung (DEGS1, n=7025) geschichtet nach Gemeindetyp

\begin{tabular}{|c|c|c|c|c|c|}
\hline & \multirow[t]{3}{*}{ Geschlecht } & \multicolumn{4}{|l|}{ Gemeindetyp } \\
\hline & & Ländlich & Kleinstädtisch & Mittelstädtisch & Großstädtisch \\
\hline & & $\%(95 \%-K I)$ & $\%(95 \%-K I)$ & $\%(95 \%-K I)$ & $\%(95 \%-K I)$ \\
\hline \multirow[t]{3}{*}{ Mindestens eine Sensibilisierung } & Frauen & $48,0(41,8-54,3)$ & $46,4(42,4-50,4)$ & $42,8(39,1-46,5)$ & $45,7(42,3-49,0)$ \\
\hline & Männer & $55,1(50,5-59,6)$ & $51,3(46,6-56,1)$ & $52,1(48,2-56,0)$ & $50,3(45,6-55,0)$ \\
\hline & Gesamt & $51,7(47,5-55,9)$ & $48,8(45,9-51,8)$ & $47,3(44,9-49,8)$ & $48,0(45,5-50,4)$ \\
\hline \multirow{3}{*}{$\begin{array}{l}\text { Sensibilisierung gegen inhalative Allergene } \\
\text { (SX1) }\end{array}$} & Frauen & $30,1(25,4-35,1)$ & $28,2(24,6-32,0)$ & $33,2(29,6-36,9)$ & $34,5(31,1-38,0)$ \\
\hline & Männer & $28,2(23,2-33,7)$ & $35,0(31,1-39,1)$ & $37,8(33,7-42,0)$ & $36,5(32,5-40,6)$ \\
\hline & Gesamt & $29,1(25,1-33,4)$ & $31,6(28,9-34,3)$ & $35,5(32,7-38,3)$ & $35,5(33,2-37,8)$ \\
\hline \multirow[t]{3}{*}{ Sensibilisierung gegen Nahrungsmittel } & Frauen & $23,9(19,3-29,0)$ & $20,6(17,6-23,7)$ & $24,6(20,8-28,7)$ & $26,7(23,1-30,5)$ \\
\hline & Männer & $24,9(21,3-28,7)$ & $26,0(21,4-30,9)$ & $28,1(24,4-32,1)$ & $27,5(24,4-30,8)$ \\
\hline & Gesamt & $24,4(21,1-27,9)$ & $23,3(20,6-26,1)$ & $26,3(24,0-28,8)$ & $27,1(24,7-29,6)$ \\
\hline \multirow[t]{3}{*}{ Sensibilisierung gegen Insektengifte } & Frauen & $24,7(20,1-29,8)$ & $25,2(21,4-29,3)$ & $14,2(11,2-17,6)$ & $16,7(13,9-19,9)$ \\
\hline & Männer & $36,4(30,9-42,1)$ & $27,7(23,0-32,7)$ & $23,5(20,1-27,0)$ & $21,7(18,3-25,4)$ \\
\hline & Gesamt & $30,8(26,2-35,6)$ & $26,4(23,1-29,9)$ & $18,7(16,3-21,3)$ & $19,2(17,0-21,5)$ \\
\hline \multirow[t]{3}{*}{ Sensibilisierung gegen Gräserpollen } & Frauen & $15,1(11,9-18,9)$ & $14,6(12,4-17,0)$ & $18,1(14,9-21,7)$ & $18,3(15,5-21,4)$ \\
\hline & Männer & $17,4(14,0-21,3)$ & $20,2(16,6-24,2)$ & $24,5(21,4-27,9)$ & $23,4(20,3-26,7)$ \\
\hline & Gesamt & $16,3(13,6-19,4)$ & $17,4(15,4-19,4)$ & $21,2(18,9-23,7)$ & $20,8(19,0-22,7)$ \\
\hline \multirow[t]{3}{*}{ Sensibilisierung gegen Baumpollen } & Frauen & $17,0(13,4-21,1)$ & $17,3(14,8-20,1)$ & $18,8(15,9-22,1)$ & $20,5(17,5-23,8)$ \\
\hline & Männer & $15,0(10,9-20,0)$ & $18,0(15,2-21,1)$ & $21,6(18,6-24,8)$ & $20,2(17,5-23,2)$ \\
\hline & Gesamt & $16,0(12,8-19,5)$ & $17,7(15,9-19,5)$ & $20,2(18,3-22,2)$ & $20,4(18,3-22,5)$ \\
\hline \multirow[t]{3}{*}{ Sensibilisierung gegen Hausstaubmilben } & Frauen & $12,1(8,9-15,8)$ & $12,6(9,9-15,7)$ & $13,9(11,3-16,8)$ & $15,6(12,9-18,6)$ \\
\hline & Männer & $13,0(9,8-16,8)$ & $16,4(13,0-20,2)$ & $20,1(16,9-23,6)$ & $19,9(16,7-23,5)$ \\
\hline & Gesamt & $12,5(10,1-15,3)$ & $14,5(12,3-16,9)$ & $16,9(14,9-19,1)$ & $17,7(15,7-19,9)$ \\
\hline \multirow[t]{3}{*}{ Sensibilisierung gegen Kräuterpollen } & Frauen & $11,9(8,2-16,4)$ & $9,3(7,4-11,5)$ & $8,6(5,9-12,1)$ & $10,7(8,4-13,3)$ \\
\hline & Männer & $9,7(6,7-13,5)$ & $13,0(9,9-16,7)$ & $12,9(10,5-15,7)$ & $13,2(11,3-15,4)$ \\
\hline & Gesamt & $10,7(8,8-12,8)$ & $11,1(9,2-13,3)$ & $10,7(8,8-12,9)$ & $11,9(10,5-13,5)$ \\
\hline \multirow[t]{3}{*}{ Sensibilisierung gegen Tierepithelien } & Frauen & $8,2(6,3-10,4)$ & $7,8(6,3-9,6)$ & $10,6(8,0-13,6)$ & $11,2(9,1-13,6)$ \\
\hline & Männer & $7,2(4,6-10,5)$ & $8,5(6,2-11,2)$ & $11,2(8,7-14,2)$ & $12,3(9,2-16,0)$ \\
\hline & Gesamt & $7,6(6,1-9,4)$ & $8,2(6,7-9,8)$ & $10,9(9,0-13,1)$ & $11,7(9,6-14,1)$ \\
\hline \multirow[t]{3}{*}{ Sensibilisierung gegen Schimmelpilze } & Frauen & $2,9(1,7-4,6)$ & $3,3(2,1-4,9)$ & $3,9(2,6-5,4)$ & $2,7(1,6-4,2)$ \\
\hline & Männer & $5,3(3,5-7,6)$ & $4,4(2,5-7,2)$ & $6,0(4,4-7,9)$ & $7,8(5,8-10,2)$ \\
\hline & Gesamt & $4,1(2,9-5,6)$ & $3,9(2,6-5,4)$ & $4,9(3,9-6,1)$ & $5,2(4,1-6,5)$ \\
\hline \multirow[t]{3}{*}{ Sensibilisierung gegen Latex } & Frauen & $5,2(3,5-7,4)$ & $3,3(2,1-5,0)$ & $3,7(2,4-5,4)$ & $3,8(2,3-5,7)$ \\
\hline & Männer & $5,8(3,9-8,2)$ & $4,8(3,3-6,7)$ & $5,3(3,8-7,3)$ & $4,6(2,9-6,7)$ \\
\hline & Gesamt & $5,5(4,2-7,0)$ & $4,1(3,1-5,2)$ & $4,5(3,4-5,8)$ & $4,2(3,1-5,4)$ \\
\hline
\end{tabular}

oder hohem sozioökonomischem Status. Dieser Unterschied war jedoch nicht statistisch signifikant. Die Prävalenz einer Sensibilisierung gegen Kräuterpollen und Latex unterschied sich ebenfalls nicht statistisch signifikant nach dem sozioökonomischen Status.

Bezogen auf die Wohnregion, zeigten sich lediglich für Inhalationsallergene, Insektengifte und Hausstaubmilben statistisch signifikante Unterschiede bei den Sensibilisierungsprävalenzen (• Tab. 4). Teilnehmer aus dem Osten Deutschlands wiesen signifikant seltener eine Sensibilisierung gegen Inhalationsallergene auf als Teilnehmer aus dem Westen. Bei Betrachtung der weitergehenden Stratifizierung des Westens zeigt sich dies nur für den Vergleich zwischen NordrheinWestfalen und der Region Ost. Teilnehmer aus Ostdeutschland waren ebenfalls seltener gegen Hausstaubmilben sensibilisiert als Teilnehmer aus Westdeutschland. Die Berücksichtigung der weitergehenden Stratifizierung von Westdeutschland zeigt, dass Teilnehmer aus dem Osten und Süden Deutschlands signifikant seltener gegen Hausstaubmilben sensibilisiert waren als die Teilnehmer aus Nordrhein-Westfalen. Hingegen wiesen Teil- nehmer aus den westlichen Bundesländern seltener Sensibilisierungen gegen Insektengifte auf als die Teilnehmer aus dem Osten. Bei Berücksichtigung der weitergehenden Stratifizierung des Westens zeigte sich, dass die Prävalenz einer Sensibilisierung gegen Insektengifte im Osten wie im Süden Deutschlands signifikant höher war als im Nordwesten und in NordrheinWestfalen. Zudem wiesen die Teilnehmer aus der Mitte Deutschlands seltener Sensibilisierungen gegen Insektengifte auf als die Teilnehmer aus dem Süden.

Die Prävalenz der Sensibilisierungen nach Gemeindetyp auf Basis der Einwoh- 


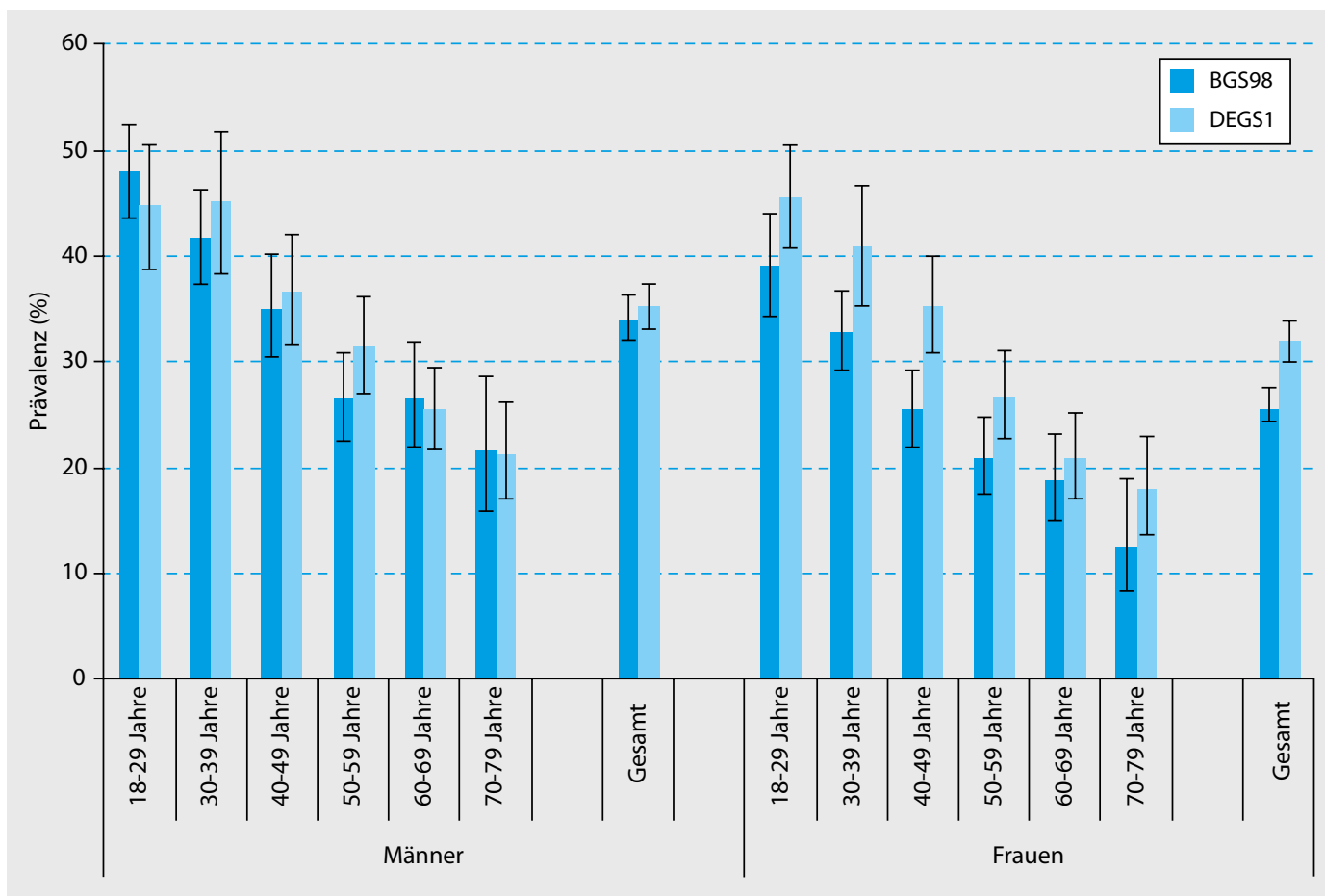

Abb. $2<$ Trend bei der Sensibilisierung gegen Inhalationsallergene (SX1) ermittelt durch Vergleich der Ergebnisse aus dem BGS98 ( $n=3271$ Männer und $n=3466$ Frauen) und aus DEGS1 ( $n=3371$ Männer und 3651 Frauen) differenziert nach Geschlecht und Alter nerzahl ist in $\bullet$ Tab. 5 dargestellt. Mit zunehmender Gemeindegröße nahm die Prävalenz von Sensibilisierungen gegen Inhalationsallergene, Nahrungsmittelallergene, Gräserpollen, Baumpollen, Hausstaubmilben und Tierepithelien zu, statistisch signifikant jedoch nur für die beiden letztgenannten Gruppen. Hingegen war die Prävalenz von Sensibilisierungen gegen Insektengifte bei Teilnehmern aus ländlichen und kleinstädtischen Gebieten signifikant höher als bei Teilnehmern aus mittelstädtischen und großstädtischen Gebieten.

\section{Trend der Sensibilisierung gegen Inhalationsallergene}

Zur Ermittlung der zeitlichen Veränderungen bei der Sensibilisierungsprävalenz wurden die Ergebnisse des SX1-Tests auf Inhalationsallergene des BGS98 mit den DEGS1-Ergebnissen verglichen. Im Vergleich zum BGS98 hat die Prävalenz einer Sensibilisierung gegen Inhalationsallergene in DEGS1 signifikant um fast 4 Prozentpunkte zugenommen: von $29,8 \%$ (95\%-Konfidenzintervall: 28,2-31,5\%) auf $33,6 \%$ (32,1-35,0\%). Bei Frauen stieg die Prävalenz statistisch signifikant um fast 7 Prozentpunkte: von 25,4\% (23,4-27,6\%) auf 32,0\% (30,1-33,9\%). Bei Männern zeigte sich dieser Trend hingegen nicht: $34,1 \%(32,0-36,3 \%)$ im BGS98 standen $35,2 \%(33,0-37,4 \%)$ im DEGS1 gegenüber (• Abb. 2). Während sich im BGS98 die Prävalenz von Sensibilisierungen gegen Inhalationsallergene signifikant zwischen Männern (34,1\%; 32,0-36,3\%) und Frauen $(25,4 \% ; 23,4-27,6 \%)$ unterschied, konnte im DEGS1 diesbezüglich kein statistisch signifikanter Unterschied mehr beobachtet werden.

\section{Diskussion}

Mit den Daten des DEGS1 kann die Prävalenz allergischer Sensibilisierungen auf repräsentativer Basis für die deutsche Erwachsenenbevölkerung im Alter von 18 bis 79 Jahren dargestellt werden. Bundesweit ist fast die Hälfte der Erwachsenen gegen mindestens eines der untersuchten Allergene sensibilisiert. In länger zurückliegenden Studien vom Anfang der 1990er-Jahre in verschiedenen Städten in West- und Ostdeutschland wurden Sensibilisierungen gegen 5 Allergene (Gräserpollen, Birkenpollen, Katzenepithelien, Hausstaubmilbe und den Schimmelpilz Cladosporium herbarum) bei Erwachsenen getestet [6, 7]. Bei den 20- bis 44-Jährigen lag die Prävalenz für eine Sensibilisierung gegen mindestens eines dieser
Allergene bei 40,3\% in Hamburg und bei $34,1 \%$ in Erfurt [7]. In einer weiteren Studie wiesen 36\% der 25- bis 64-Jährigen aus Augsburg und 26\% der 25- bis 64-Jährigen aus Erfurt eine Sensibilisierung gegen mindestens eines der 5 oben genannten Allergene auf [6]. Im Kinder- und Jugendgesundheitssurvey (KiGGS) aus den Jahren 2003 bis 2006 wurden Tests auf $20 \mathrm{Al}-$ lergene durchgeführt. Insgesamt wiesen $40,8 \%$ der 3- bis 17-Jährigen in Deutschland eine Sensibilisierung gegen mindestens eines der 20 getesteten Einzelallergene auf. Bei den 14- bis 17-Jährigen lag die Prävalenz einer Sensibilisierung sogar bei 46,6\% [26]. Im US-amerikanischen Gesundheitssurvey „National Health and Nutrition Examination Survey (NHANES) 2005-2006“ wurden Tests auf 19 Allergene in einer bevölkerungsbezogenen Stichprobe ab einem Alter von 6 Jahren durchgeführt. Die Prävalenz für eine Sensibilisierung gegen mindestens eines der untersuchten Allergene lag bei 43,7\% [27].

Nach den Ergebnissen des DEGS1 ist die Prävalenz für Sensibilisierungen gegen Nahrungsmittelallergene bei Erwachsenen in Deutschland beträchtlich. Insbesondere waren deutsche Erwachsene häufig gegen pollenassoziierte Nahrungsmittelallergene sensibilisiert, d. h. gegen Nahrungsmittelallergene, die mit IgE-Anti- 
körpern gegen Baum-, Kräuter- oder Gräserpollen kreuzreagieren können, wie Haselnuss, Soja, Erdnuss und grüner Apfel. In einer Studie bei Erwachsenen im Alter von 25 bis 74 Jahren aus der Region Augsburg wurde 1994/1995 das Auftreten von Sensibilisierungen gegen $10 \mathrm{Nah}-$ rungsmittelallergene mittels Hautpricktests untersucht. Demnach war die Prävalenz der Sensibilisierung gegen mindestens eines der untersuchten Nahrungsmittelallergene mit $16,8 \%$ ebenfalls hoch, jedoch geringer als im DEGS1 [28]. Dieser Unterschied zwischen DEGS1 und der Augsburger Studie kann zum Teil auf die Zahl der untersuchten Nahrungsmittelallergene zurückgeführt werden. Auch in der Augsburger Studie wiesen die Teilnehmer am häufigsten Sensibilisierungen gegen pollenassoziierte Nahrungsmittelallergene auf. Zudem traten Sensibilisierungen gegen Nahrungsmittelallergene häufig gleichzeitig mit Sensibilisierungen gegen Inhalationsallergene auf, was einen hohen Anteil an Kreuzsensibilisierungen vermuten lässt, Ko-Sensibilisierungen aber nicht ausschließt [28].

Ein erheblicher Anteil der deutschen Erwachsenen ist gegen Insektengifte sensibilisiert. Die in der vorliegenden Studie ermittelte diesbezügliche Prävalenz ist mit den Ergebnissen bisher durchgeführter Studien in der Allgemeinbevölkerung in Europa (9-29\%) und in einzelnen Regionen Deutschlands (ländliche Region Bayern 27\% und Hamburg 25\%) vergleichbar [2, 29]. Die Prävalenz für Sensibilisierungen gegen Insektengifte ist abhängig von der Wahrscheinlichkeit der Exposition [29]. Damit können die regionalen Unterschiede und die Unterschiede nach Gemeindetyp, die in DEGS1 beobachtet wurden, wenigstens zum Teil erklärt werden. Daraus erklärt sich auch die Altersunabhängigkeit der Prävalenz bzw. ihr leichter Anstieg bei höheren Altersgruppen. Vertiefende Analysen sollen klären, inwieweit auch hier Kreuzreaktionen einen Teil der hohen Prävalenzen erklären können.

Die Ergebnisse des DEGS1 unterstreichen auch die Bedeutung der Sensibilisierungen gegen Gräser-, Baum- und Kräuterpollen. Im Zuge von Klimaveränderungen hat sich die Pollenflugzeit von Gräserund Baumpollen in den letzten Jahrzehn- ten verlängert [30,31]. Demzufolge hat die Expositionsdauer gegenüber Pollen zugenommen, was sich möglicherweise auf die Sensibilisierungsprävalenzen ausgewirkt hat. Von besonderem Interesse ist die rasante Verbreitung der aus Nordamerika stammenden hoch-allergenen Ambrosia in Deutschland [30, 31]. DEGS1 zeigt, dass rund $11 \%$ der Erwachsenen in Deutschland gegen Kräuterpollen, zu denen auch die Pollen der Gattung Ambrosia gehören, sensibilisiert sind. Die im DEGS1 ermittelten Prävalenzen für die 3 untersuchten Ambrosia-Arten sind vergleichbar mit in der Schweiz ermittelten Prävalenzen [32]. Erste Zwischenauswertungen der Daten des DEGS1 deuten auf eine hohe Kreuzreaktivität von Beifuß- und Ambrosiapollen hin [31].

In bisherigen Studien wurden regionale Unterschiede bei den Sensibilisierungsprävalenzen in Deutschland oft als Ost-West-Unterschiede dargestellt $[6,7,9$, 11]. Die vorliegenden Auswertungen zeigen, dass Ost-West-Vergleiche die regionalen Unterschiede in den Sensibilisierungsprävalenzen in Deutschland nicht ausreichend beschreiben. Im DEGS1 wurden neben Ost-West-Unterschieden auch Nord-Süd-Unterschiede in den Sensibilisierungsprävalenzen gezeigt. Die Beschreibung der Ost-West-Unterschiede erlaubt jedoch einen Vergleich mit Ergebnissen vorangegangener Studien. In Übereinstimmung mit diesen Studien wurde beispielsweise in DEGS1 gezeigt, dass die Prävalenz von Sensibilisierungen gegen Hausstaubmilben in Westdeutschland höher war als in Ostdeutschland [7, 8]. Genauer betrachtet wiesen Erwachsene aus Nordwestdeutschland etwas häufiger und aus Nordrhein-Westfalen signifikant häufiger Sensibilisierungen gegen Hausstaubmilben auf als Erwachsene aus dem Süden und Osten Deutschlands. Dies kann möglicherweise mit klimatischen Unterschieden (feuchteres Klima im Nordwesten vs. trockeneres Klima im Osten und Süden) zusammenhängen.

Darüber hinaus wurden in DEGS1 teilweise Unterschiede in der Prävalenz von Sensibilisierungen nach Gemeindetyp beobachtet, die möglicherweise mit Unterschieden in den Wohnverhältnissen (z. B. unterschiedliche Schadstoffbelastungen) erklärt werden könnten.
Sensibilisierungen gegen Schimmelpilze sind selten. Dieses Ergebnis ist positiv zu bewerten, da diese Sensibilisierungen gegen Schimmelpilze einen bedeutenden Risikofaktor für Asthma bronchiale darstellt $[33,34,35]$.

Am seltensten sind Sensibilisierungen gegen Latex. Es wird davon ausgegangen, dass vor allem bestimmte Berufsgruppen, z. B. Mitarbeiter im Gesundheitswesen, von einer Latexallergie betroffen sind [2].

Die im DEGS1 beobachteten Unterschiede in den Sensibilisierungsprävalenzen nach Alter und Geschlecht finden sich auch in anderen Studien $[4,6,7,9]$. So konnte auch im DEGS1 gezeigt werden, dass Männer gegen Insektengifte, Gräserpollen, Hausstaubmilben und Schimmelpilze statistisch signifikant häufiger sensibilisiert sind als Frauen. Die Unterschiede können möglicherweise mit einer unterschiedlich hohen Expositionsstärke und -dauer gegenüber Allergenen zusammenhängen. Andererseits können auch geschlechtsspezifische Unterschiede bei der Immunreaktion eine Rolle spielen.

Der positive Zusammenhang zwischen vorliegenden Sensibilisierungen und höherem sozioökonomischem Status steht ebenfalls im Einklang mit den Ergebnissen aus anderen Studien $[8,11,26]$. Dieser kann möglicherweise durch Unterschiede in den Lebensbedingungen erklärt werden. Strachan diskutierte 1989 zum ersten Mal den protektiven Effekt des Kontakts mit Mikroorganismen, die sog. HygieneHypothese [36].

\section{Fazit und Ausblick}

Die dargestellten ersten Ergebnisse von DEGS1 zeigen, dass viele deutsche Erwachsene von allergischen Sensibilisierungen betroffen sind. In den vergangenen 10 Jahren hat die Prävalenz von Sensibilisierungen gegen Inhalationsallergene zugenommen. In vertiefenden Auswertungen sollen die Zusammenhänge zwischen dem Auftreten von Sensibilisierungen und allergischen Erkrankungen untersucht werden. Zudem soll die Problematik der Kreuzreaktivität von Allergenen Thema vertiefender Auswertungen sein. Bisher gibt es nur wenige Studien, die die individuellen Veränderungen im 
Sensibilisierungsstatus über die Zeit beschrieben haben. Aus diesem Grund sind zur Auswertung der Sensibilisierung gegen Inhalationsallergene Längsschnittanalysen für Personen geplant, die sowohl am BGS98 als auch am DEGS1 teilgenommen haben.

\section{Korrespondenzadresse}

\section{Haftenberger}

Abteilung für Epidemiologie und Gesundheitsmonitoring, Robert Koch-Institut General-Pape-Str. 62-66, 12101 Berlin haftenbergerm@rki.de

Finanzierung der Studie. Die Studie zur Gesundheit Erwachsener in Deutschland wurde finanziert mit Mitteln des Robert Koch-Instituts und des Bundesministeriums für Gesundheit. Die Förderung des „Allergie- und Sensibilisierungsmonitorings im Rahmen der nationalen Gesundheitssurveys des Robert Koch-Instituts (RKI) zur Einschätzung der Allergiegefährdung der erwachsenen Bevölkerung" erfolgte aus Mitteln des Bundesministeriums für Ernährung, Landwirtschaft und Verbraucherschutz (BMELV) über die Bundesanstalt für Landwirtschaft und Ernährung (BLE).

Interessenkonflikt. Der korrespondierende Autor gibt für sich und seine Koautoren an, dass kein Interessenkonflikt besteht

\section{Literatur}

1. Asher MI, Montefort S, Bjorksten B et al (2006) Worldwide time trends in the prevalence of symptoms of asthma, allergic rhinoconjunctivitis, and eczema in childhood: ISAAC phases one and three repeat multicountry cross-sectional surveys. Lancet 368:733-743

2. DGAI/ÄDA/DAAU/GPA (2010) Weißbuch Allergie in Deutschland. Springer Medizin, Urban \& Vogel $\mathrm{GmbH}$, München

3. Langen U, Schmitz R, Steppuhn H (2013) Häufigkeit allergischer Erkrankungen in Deutschland. Ergebnisse aus der Studie zur Gesundheit Erwachsener in Deutschland (DEGS1). Bundesgesundheitsbl Gesundheitsforsch Gesundheitsschutz 56:698-707

4. Hermann-Kunz E, Thierfelder W (2001) Allergische Rhinitis und Sensibilisierungsraten - Nimmt die Prävalenz wirklich zu. Wie sicher sind unsere Informationen. Bundesgesundheitsbl Gesundheitsforsch Gesundheitsschutz 44:643-653

5. Langen U (2012) Classification of specific lgE antibodies in children with hay fever and other atopic diseases in Germany. Results of the German Health Interview and Examination Survey for Children and Adolescents (KiGGS). Bundesgesundheitsbl Gesundheitsforsch Gesundheitsschutz 55:318-328

6. Filipiak B, Heinrich J, Nowak D, Wichmann HE (2001) The distribution in specific lgE and the prevalence of allergic symptoms in 25-64-years old inhabitants of an eastern and a western German city - results from Augsburg and Erfurt. Eur J Epidemiol 17:77-84
7. Nowak D, Heinrich J, Jorres R et al (1996) Prevalence of respiratory symptoms, bronchial hyperresponsiveness and atopy among adults: west and east Germany. Eur Respir J 9:2541-2552

8. Schafer T, Ruhdorfer S, Weigl L et al (2001) School education and allergic sensitization in adults. Allergy 56:1206-1210

9. Heinrich J, Richter K, Frye C et al (2002) European Community Respiratory Health Survey in adults (ECRHS). Pneumologie 56:297-303

10. Linneberg A, Gislum M, Johansen N et al (2007) Temporal trends of aeroallergen sensitization over twenty-five years. Clin Exp Allergy 37:1137-1142

11. Nicolai T, Bellach B, Mutius EV et al (1997) Increased prevalence of sensitization against aeroallergens in adults in West compared with East Germany. Clin Exp Allergy 27:886-892

12. Krämer U (2006) Die niedrige Prävalenz spezifischer IgE-Senisbilisierungen bei älteren Personen ist ein Kohortenerffekt - Ergebnisse der Bundesgesundheitssurveys 1990/1992 und 1997/1998. Kolloquium im Robert Koch-Insitut

13. Kurth $B M$, Lange $C$, Kamtsiuris $P$, Hölling H (2009) Gesundheitsmonitoring am Robert Koch-Institut. Bundesgesundheitsbl Gesundheitsforsch Gesundheitsschutz 52:557-570

14. Kurth BM (2012) Das RKI-Gesundheitsmonitoring - was es enthält und wie es genutzt werden kann Public Health Forum 20(76):4.e1-4.e3

15. Gößwald A, Lange M, Kamtsiuris P, Kurth BM (2012) DEGS: Studie zur Gesundheit Erwachsener in Deutschland. Bundesweite Quer- und Längsschnittstudie im Rahmen des Gesundheitsmonitorings des Robert Koch-Instituts. Bundesgesundheitsbl Gesundheitsforsch Gesundheitsschutz 55:775-780

16. Scheidt-Nave C, Kamtsiuris $P$, Gößwald A et al (2012) German Health Interview and Examination Survey for Adults (DEGS) - design, objectives and implementation of the first data collection wave (DEGS1). BMC Public Health 12:730

17. Kamtsiuris $P$, Lange $M$, Hoffmann R et al (2013) Die erste Welle der Studie zur Gesundheit Erwachsener in Deutschland (DEGS1). Stichprobendesign, Response, Gewichtung und Repräsentativität. Bundesgesundheitsbl Gesundheitsforsch Gesundheitsschutz 56:620-630

18. Robert Koch-Institut (Hrsg) (2009) DEGS - Studie zur Gesundheit Erwachsener in Deutschland. Projektbeschreibung. Beiträge zur Gesundheitsberichterstattung des Bundes. RKI, Berlin

19. Gößwald A, Lange M, Dölle R, Hölling H (2013) Die erste Welle der Studie zur Gesundheit Erwachsener in Deutschland (DEGS1). Gewinnung von Studienteilnehmenden, Durchführung der Feldarbeit und Qualitätsmanagement. Bundesgesundheitsbl Gesundheitsforsch Gesundheitsschutz 56:611-619

20. Lampert T, Kroll L, Müters S, Stolzenberg H (2013) Messung des sozioökonomischen Status in der Studie zur Gesundheit Erwachsener in Deutschland (DEGS1). Bundesgesundheitsbl Gesundheitsforsch Gesundheitsschutz 56:631-636

21. R-Developement-Core-Team (2010) R: a language and environment for statistical computing. $R$ foundation for statistical computing. Vienna

22. Lumley T (2011) Survey analysis of complex samples. R package version 3.26

23. Lumley T (2010) Complex surveys. A guide to analysis using R. Wiley, New Jersey

24. Korn EL, Graubard BI (1998) Confidence intervals for proportions with small expected number of positive counts estimated from survey data. Surv Methodol 23:193-201
25. Falissard B (2012) Analysis of questionnaire data with R. Chapman and Hall (Hrsg), CRC-Press Taylor and Francis Group, Boca Raton

26. Schlaud M, Atzpodien K, Thierfelder W (2006) Allergische Erkrankungen. Ergebnisse aus dem Kinder- und Jugendgesundheitssurvey (KiGGS). Bundesgesundheitsbl Gesundheitsforsch Gesundheitsschutz 50:701-710

27. Salo PM, Calatroni A, Gergen PJ et al (2011) Allergy-related outcomes in relation to serum lgE: results from the National Health and Nutrition Examination Survey 2005-2006. J Allergy Clin Immunol 127:1226-1235.e1227

28. Schafer T, Bohler E, Ruhdorfer $S$ et al (2001) Epidemiology of food allergy/food intolerance in adults: associations with other manifestations of atopy. Allergy 56:1172-1179

29. Schafer T, Przybilla B (2009) Epidemiologie der Insektengiftallergie. Allergo J 18:353-358

30. D'Amato G, Cecchi L, Bonini S et al (2007) Allergenic pollen and pollen allergy in Europe. Allergy 62:976-990

31. Eis D, Helm D, Laußmann D, Stark K (2010) Klimawandel und Gesundheit - ein Sachstandsbericht. Robert Koch-Institut, Berlin

32. Ackermann-Liebrich U, Schindler C, Frei P et al (2009) Sensitisation to Ambrosia in Switzerland: a public health threat on the wait. Swiss Med Wkly 139:70-75

33. Laussmann D, Haftenberger $M$, Langen $U$, Eis $D$ (2012) Determinants of asthma among children and adolescents in Germany. Results of the German Health and Examination Survey for Children and Adolescents (KiGGS). Bundesgesundheitsbl Gesundheitsforsch Gesundheitsschutz 55:308-317

34. Schmitz R, Atzpodien K, Schlaud M (2012) Prevalence and risk factors of atopic diseases in German children and adolescents. Pediatr Allergy Immunol 23:716-723

35. Zureik M, Neukirch C, Leynaert B et al (2002) Sensitisation to airborne moulds and severity of asthma: cross sectional study from European Community Respiratory Health Survey. BMJ 325:411414

36. Strachan DP (1989) Hay fever, hygiene, and household size. BMJ 299:1259-1260 